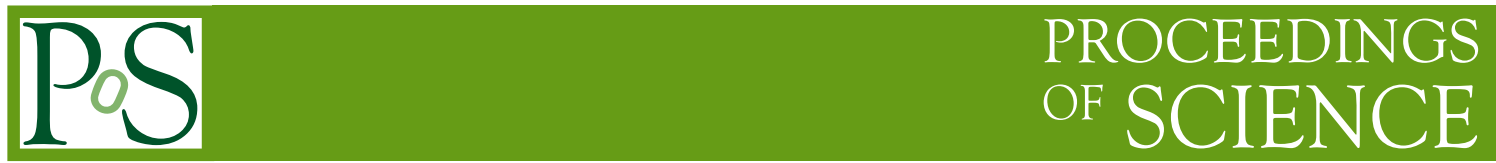

\title{
What we have learned about the Higgs boson from the bosonic decay channels and more inclusive combinations of data
}

\section{Li Yuan*, on behalf of the ATLAS and CMS collaborations}

Kobe University, Kobe, Japan

E-mail: li.yuandcern.ch; lyuandihep.ac.cn

\begin{abstract}
A review on the property measurements of the Higgs boson is presented in this report, especially on the measurements using bosonic decay channels from both ATLAS and CMS experiments at the Large Hadron Collider. The results include mass, spin/CP, couplings as well as the fiducial differential cross section measurements.
\end{abstract}

The European Physical Society Conference on High Energy Physics 22-29 July 2015

Vienna, Austria

\footnotetext{
* Speaker.
} 


\section{Introduction}

The successful Run 1 data taking at the Large Hadron Collider (LHC) provided high quality data with $\sim 5 \mathrm{fb}^{-1}$ at $\sqrt{s}=7 \mathrm{TeV}$ and $\sim 20 \mathrm{fb}^{-1}$ at $\sqrt{s}=8 \mathrm{TeV}$ for both the ATLAS [1] and CMS [2] experiments. The integrated luminosity of full Run 1 data at $\sqrt{s}=8 \mathrm{TeV}$ is about 3 times of those used at the time of the discovery of the Higgs boson [3, 4], thus the analysis based on full data consolidates the observation and allows precision measurement of the Higgs boson properties. This report summarizes the legacy results of the final analyses of the full Run 1 data from ATLAS and CMS experiments on the property measurements of the Higgs boson, including mass, spin/CP, couplings and the fiducial differential cross sections.

Among the five main decay channels to probe the presence of the Higgs boson, the bosonic channels show much better sensitivity, as can be seen in Table 1. The first two channels $(\gamma \gamma$ and $Z Z^{*} \rightarrow 4 l$ ) have the best sensitivity and significantly benefit from the excellent mass resolution. This provides the means to reconstruct a narrow mass peak of the Higgs boson. The relative mass resolutions $\left(\sigma_{m} / m_{H}\right)$ in these two channels are at the level of $1 \sim 2 \%$. There is no mass peak in $W W^{*} \rightarrow l v l v$ channel due to the final state neutrinos. However, the relatively large expected yield from this channel compensates the sensitivity. The Higgs boson signal has been independently observed by ATLAS and CMS experiments in almost every bosonic channel.

Table 1: Observed (expected) Higgs signal significance in the main decay channels. Numbers marked with $\dagger$ assume $m_{H}=125 \mathrm{GeV}$.

\begin{tabular}{c|c|c}
\hline \hline Significance obs.(exp.) & ATLAS $m_{H}=125.36 \mathrm{GeV}$ & CMS $m_{H}=125.6 \mathrm{GeV}$ \\
\hline \hline$H \rightarrow \gamma \gamma$ & $5.2 \sigma(4.6 \sigma)$ & $5.7 \sigma(5.2 \sigma)$ \\
$H \rightarrow Z Z^{*} \rightarrow 4 l$ & $8.1 \sigma(6.2 \sigma)$ & $6.8 \sigma(6.7 \sigma)$ \\
$H \rightarrow W W^{*} \rightarrow l v l \nu$ & $6.1 \sigma(5.8 \sigma)$ & $4.3 \sigma(5.8 \sigma)$ \\
$H \rightarrow \tau \tau$ & $4.5 \sigma(3.4 \sigma)$ & $3.2 \sigma(3.7 \sigma)^{\dagger}$ \\
$H \rightarrow b b$ & $1.4 \sigma(2.6 \sigma)$ & $2.1 \sigma(2.1 \sigma)^{\dagger}$ \\
\hline \hline
\end{tabular}

\section{Mass}

In the Standard Model (SM), the mass of the Higgs boson is not predicted. If the mass is known, all Higgs couplings including production and decay within the SM can be derived. The good mass resolutions of $\gamma \gamma$ and $Z Z^{*} \rightarrow 4 l$ channels rely on the good energy and direction measurement from the final state objects (photons or leptons). In both ATLAS and CMS experiments, the energy scale uncertainty for the leptons (electrons or muons) is well controlled within 1\%o level. The energy reconstruction is more challenging for photons, especially for those photons already converted into electron-positron pairs before reaching the calorimeter. Both experiments can still control the energy scale uncertainty for photons at a few \%o level.

In the $H \rightarrow \gamma \gamma$ channel, the ATLAS experiment has specific analysis strategy which divides the selected dataset into 10 categories based on the photon conversions, $\eta$ positions as well as the di-photon system $\mathrm{p}_{\mathrm{T}}$ in order to maximize the sensitivity on the mass measurement [5]. The CMS 
experiment uses the common analysis strategy as the coupling measurement in this channel, which divides the selected dataset into 14 categories based on the production modes [6].

The $H \rightarrow Z Z^{*} \rightarrow 4 l$ channel has a rather low branching ratio, thus the statistics is rather low. Here the clean signature with four leptons in the final state helps to greatly suppress the background and provide a good signal over background ratio. Both ATLAS [5] and CMS [7] experiments have performed a multidimensional fit on the $4 l$ invariant mass and other kinematic variables (or BDT trained from several kinematic variables) to extract the Higgs boson mass for this channel.

A combined LHC mass measurement [8] was performed by combining $\gamma \gamma$ and $Z Z^{*} \rightarrow 4 l$ channels and combining ATLAS and CMS experiments. The measured mass is $m_{H}=125.09 \pm$ 0.21 (stat.) \pm 0.11 (scale) \pm 0.02 (other) \pm 0.01 (theory) $\mathrm{GeV}$. The result is still statistically limited.

\section{Spin/CP}

The spin/CP of the Higgs boson in the SM is predicted to be $J^{C P}=0^{++}$. Beyond SM (BSM) theories could predict the boson with other states of spin or $\mathrm{CP}$, or even a mixture of $\mathrm{CP}$-even and $\mathrm{CP}$-odd states. At the LHC experiments, many alternative hypotheses of spin/CP states could be tested. Based on the full Run 1 dataset, the studies are only plausible with bosonic channels due to their good enough sensitivities to the presence of the Higgs boson.

In the ATLAS experiment, the hypotheses are based on Effective Field Theory [9], which assumes a general effective Lagrangian compatible with Lorentz invariance. In contrast, in the CMS experiment, the spin/CP models are based on anomalous coupling approach [10], which assumes the general amplitude compatible with the Lorentz and gauge invariance. The discriminants in $H \rightarrow Z Z^{*} \rightarrow 4 l$ channel include the decay angles of the final states leptons, the invariant masses of the two di-lepton systems as well as the invariant mass of four leptons system. In the $H \rightarrow W W^{*} \rightarrow l v l v$ channel, kinematic variables of the di-lepton system, such as $\Delta \phi_{11}, \mathrm{p}_{11}^{\mathrm{T}}$ and $\mathrm{m}_{11}$, are sensitive to the spin/CP studies. In the $H \rightarrow \gamma \gamma$ channel, spin- 1 is forbidden by the Laudau-Yang theorem, thus only spin-2 hypotheses are tested in this channel.

A combined study for spin/CP measurement in the three bosonic channels has been performed in the ATLAS experiment [11]. Results show that data strongly favors the SM hypothesis, which is a CP-even scalar boson, while all the tested alternative hypotheses other than the SM hypothesis are excluded at more than $99.9 \%$ confidence level, as shown in the left plot of Figure 1, Similar study is also performed in the CMS experiment [12]. The alternative hypotheses under test are also mostly excluded at the confidence interval over $99.9 \%$, shown in the right plot of Figure 1

\section{Couplings}

The Higgs boson coupling measurement is an important test of the Higgs sector and sensitive to the new phenomena, such as BSM coupling to Higgs sector. The coupling measurement in LHC experiments assumes no change on the tensor structure but only the coupling strength, i.e assuming the Higgs boson to be a CP-even scalar. The deviation in the observed and predicted rates is tested using the signal strength, defined as $\mu=\frac{N^{\text {observedHigg }}}{N^{\text {expectedHiggs }}}=\frac{(\sigma \cdot B R)}{(\sigma \cdot B R)_{S M}}$. It can also be interpreted as a change in coupling strengths between the Higgs boson and the involved particles. 

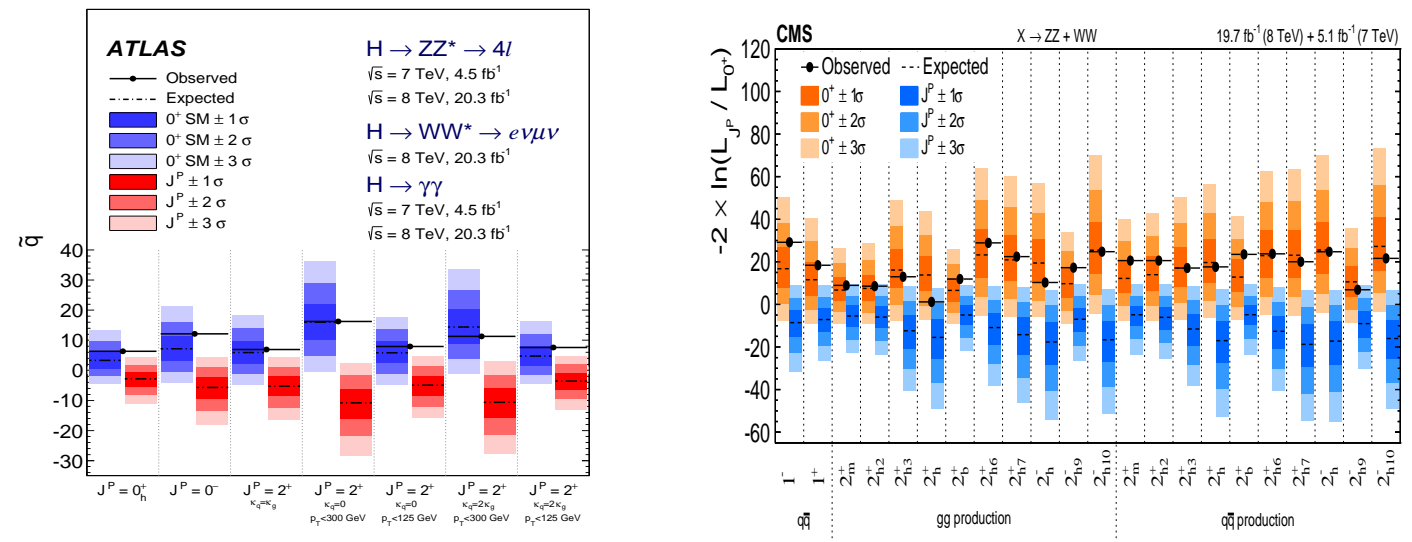

Figure 1: Left: Distributions of the test statistic q for the SM Higgs boson (blue) and for the alternative spin/CP hypotheses (red) in the ATLAS experiment. The expected median (black dashed line) and the \pm 1 , $\pm 2, \pm 3 \sigma$ regions are shown. Black points are the observed q value. Plot from Ref. [11]. Right: similar results in the CMS experiment with different color convention: the SM Higgs boson (orange), alternative hypotheses (blue), data points (black points). Plot from Ref. [12].

In order to maximize the sensitivity for the different Higgs production processes and to probe the Higgs coupling to bosons and fermions in the different bench-mark models, a comprehensive and inclusive analysis by combining the main decay channels (bosonic and fermionic channels) and the rare production or decay channels (such as $t \bar{t} H$ production, and $H \rightarrow Z \gamma$ or $H \rightarrow$ invisible decays) is performed by both ATLAS [13] and CMS [14] experiments.

A simple test of compatibility with the SM was performed by measuring the combined signal strength, which assumes all the production and decay channels share the same signal strength. In this test, ATLAS experiment measures the combined signal strength to be $\mu_{\text {combined }}=1.18_{-0.14}^{+0.15}$, and CMS experiment gets $\mu_{\text {combined }}=1.00 \pm 0.14$. In Figure 2, one can find the measured signal strength for each production mode from ATLAS (left) and CMS (right) experiments. Figure 3 show the measured signal strength for each decay channel from ATLAS (left) and CMS (right) experiments. Another test to probe the Higgs coupling to fermions and bosons was done by assuming all the fermions share the same coupling strength $\left(\kappa_{F}\right)$ while all the vector bosons share the same coupling strength $\left(\kappa_{V}\right)$. In this test, ATLAS experiment obtained the best fit values of $\kappa_{V}=1.09_{-0.07}^{+0.07}, \kappa_{F}=1.11_{-0.15}^{+0.17}$. The corresponding values from CMS experiment are $\kappa_{V}=1.01_{-0.07}^{+0.07}, \kappa_{F}=0.87_{-0.13}^{+0.14}$. All the measured signal strengths and other tests on Higgs couplings, summarized in references [13, 14], show consistent results with the SM prediction. No evidence of a significant deviation from SM is observed.

\section{Cross sections}

The total and differential Higgs production cross sections are measured by the LHC experiments. The measurements are performed for $\gamma \gamma$ and $Z Z^{*} \rightarrow 4 l$ channels and later combined [15]. The total cross section measured from the two channels agree well with each other; their combined measurement gives $\sigma=33.0 \pm 5.3$ (stat.) $\pm 1.6($ syst.) $\mathrm{pb}$, which is slightly larger than the SM 

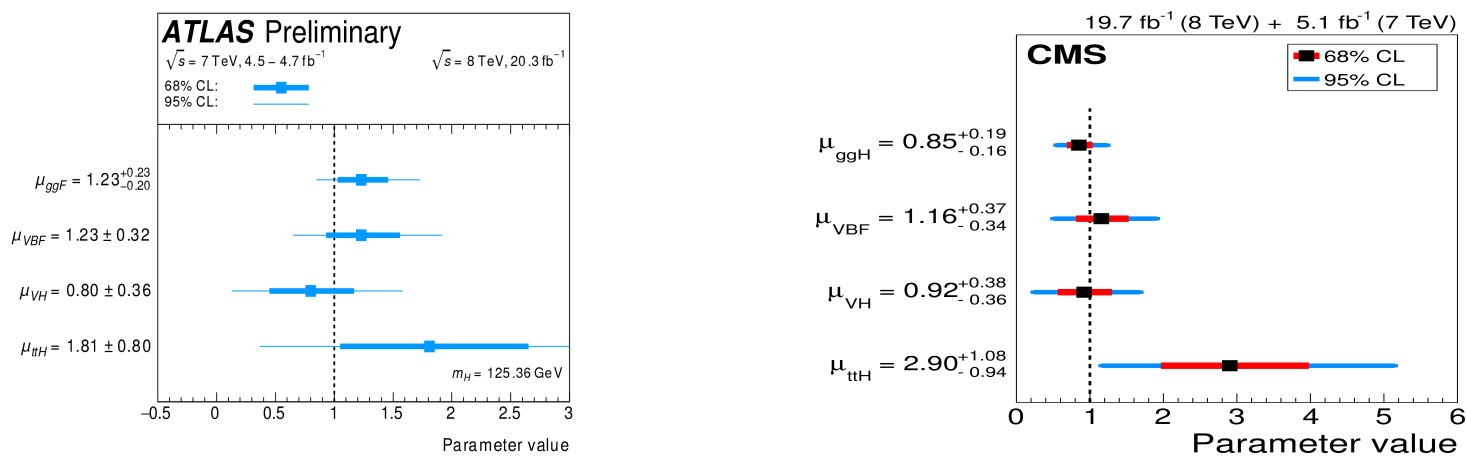

Figure 2: Left: measured signal strength for each Higgs production channel in the ATLAS experiment. Plot from Ref. [13]. Right: measured signal strength for each Higgs production channel in the CMS experiment. Plot from Ref. [14].
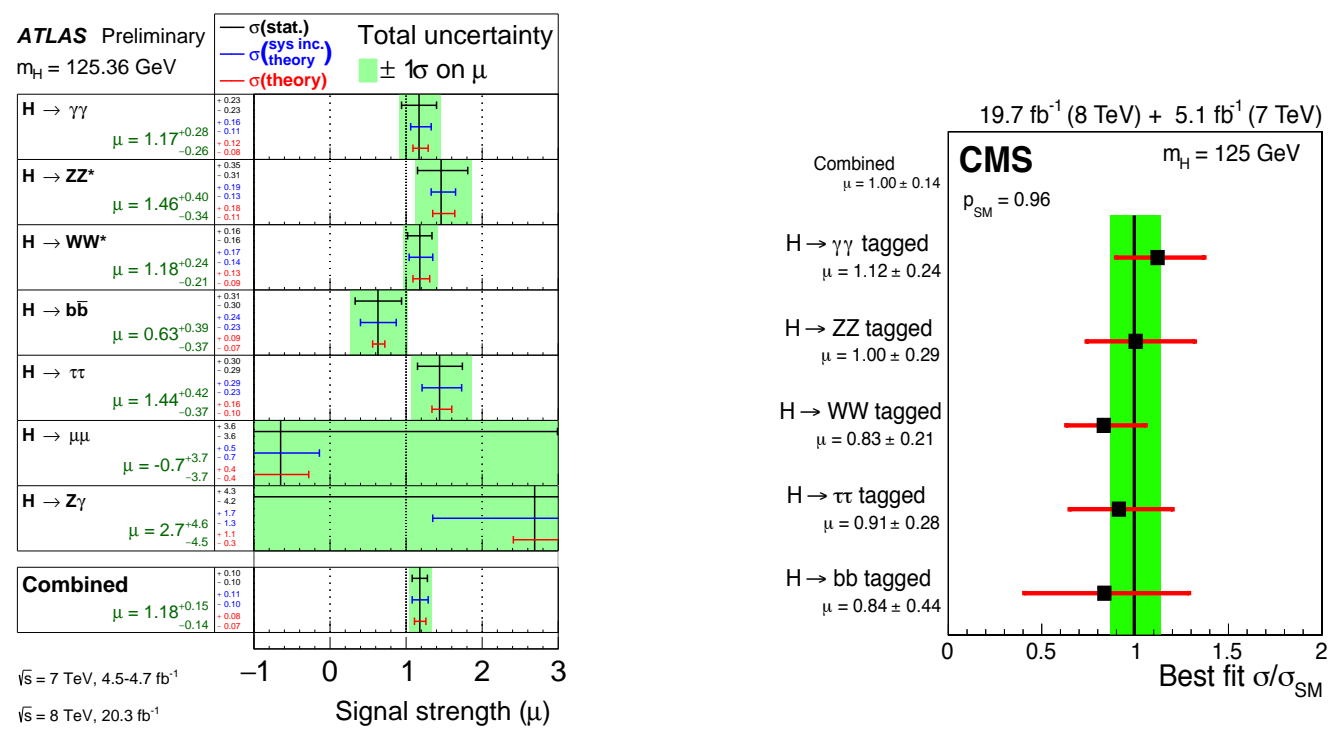

Figure 3: Left: measured signal strength for each Higgs decay channel in ATLAS experiment. Plot from Ref. [13]. Right: measured signal strength for each Higgs decay channel in CMS experiment. Plot from Ref. [14].

prediction but not deviating significantly with respect to the estimated uncertainties. The Higgs differential cross sections of several kinematic variables have been measured, including the associated jet multiplicity, transverse momentum and rapidity of the Higgs boson, as well as transverse momentum of the leading associated jet in the event. Results in Figure 4 show data slightly prefers a more boosted Higgs boson (i.e harder Higgs boson $\mathrm{p}_{\mathrm{T}}$ spectrum) and more associated jets. However, the results still have very large statistical uncertainties.

\section{Summary}

The full Run 1 data at the LHC has been fully exploited to measure the Higgs boson properties. The Higgs mass is determined to be $m_{H}=125.09 \pm 0.24 \mathrm{GeV}$. The data strongly favors the Higgs boson to be CP-even scalar. The Higgs couplings and differential cross sections are consistent 

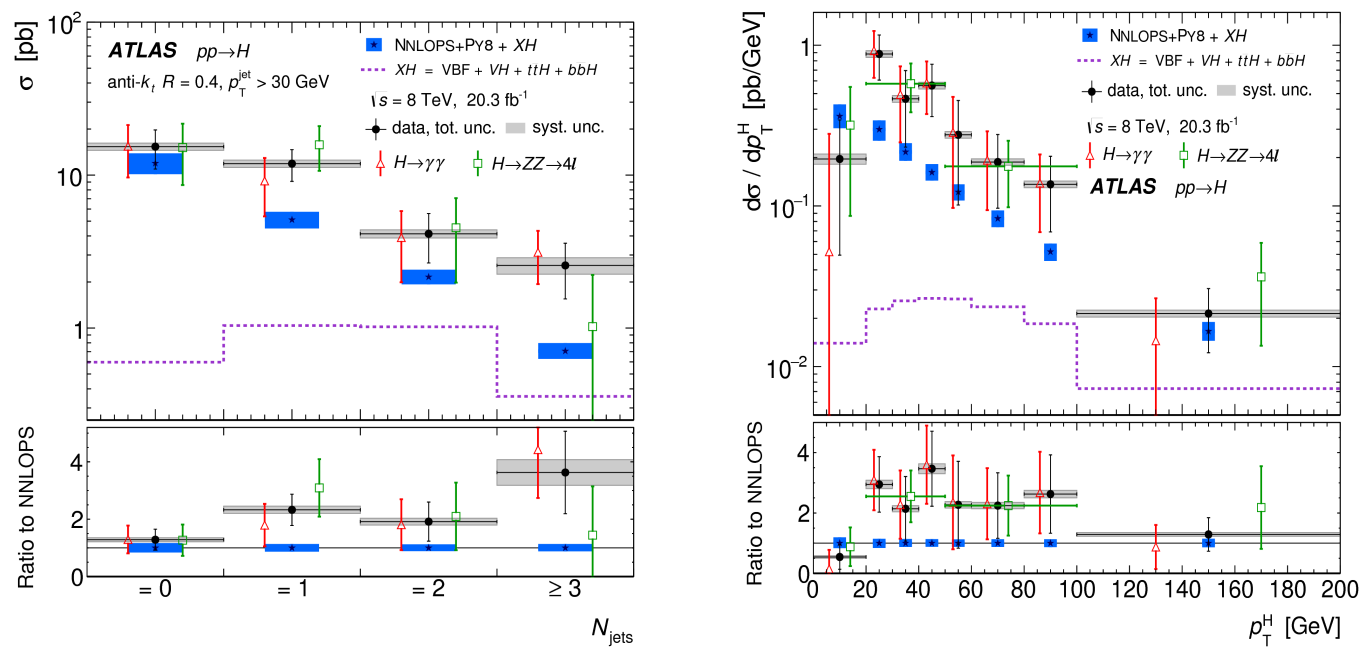

Figure 4: Left: measured Higgs differential cross section as a function of associated jet multiplicity. Right: measured Higgs differential cross section as a function of Higgs transverse momentum. Plots from Ref. [15].

with the SM prediction, no significant deviation is observed so far, although results are rather statistically limited. The LHC Run 2 with higher energy has already started and is expected to deliver five times more integrated luminosity than Run 1. The Higgs production cross sections in Run 2 will increase by a factor of 2.1 2.6 for the main production channels due to the increased center of mass energy. Main backgrounds will also increase by similar factors. More precise Higgs property measurements are foreseen in Run 2.

\section{References}

[1] ATLAS Collaboration, 2008 JINST 3 S08003.

[2] CMS Collaboration, 2008 JINST 3 S08004.

[3] ATLAS Collaboration, Phys. Lett. B 716 (2012) 1, [arXiv:1207.7214].

[4] CMS Collaboration, Phys. Lett. B 716 (2012) 30, [arXiv:1207.7235].

[5] ATLAS Collaboration, Phys. Rev. D 90 (2014) 052004, [arXiv:1406.3827].

[6] CMS Collaboration, Eur. Phys. J. C 74 (2014) 3076, [arXiv:1407.0558].

[7] CMS Collaboration, Phys. Rev. D 89 (2014) 092007, [arXiv:1312.5353].

[8] ATLAS and CMS Collaborations, Phys. Rev. Lett. 114 (2015) 191803, [arXiv:1503.07589].

[9] P. Artoisenet et al., JHEP 1311 (2013) 043, [arXiv:1306.6464].

[10] S. Bolognesi et al., Phys. Rev. D 86 (2012) 095031, [arXiv:1208.4018].

[11] ATLAS Collaboration, arXiv:1506.05669.

[12] CMS Collaboration, Phys. Rev. D 92 (2015) 012004, [arXiv:1411.3441].

[13] ATLAS Collaboration, arXiv:1507.04548.

[14] CMS Collaboration, Eur. Phys. J. C 75 (2015) 212, [arXiv:1412.8662].

[15] ATLAS Collaboration, Phys. Rev. Lett. 115 (2015) 091801, [arXiv:1504.05833]. 